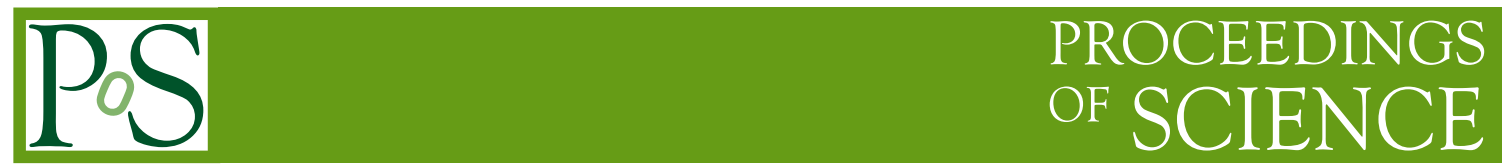

\title{
Three Dimensional $N=2$ Supersymmetry on the Lattice
}

Joshua W. Elliott*

McGill University

E-mail: elliottj@hep.physics.mcgill.ca

\section{Guy D. Moore}

McGill University

E-mail: guymoore@hep.physics.mcgill.ca

We show how 3-dimensional, $N=2$ supersymmetric theories, including super QCD with matter fields, can be put on the lattice with existing techniques, in a way which will recover supersymmetry in the small lattice spacing limit. Residual supersymmetry breaking effects are suppressed in the small lattice spacing limit by at least one power of the lattice spacing $a$.

XXIIIrd International Symposium on Lattice Field Theory

25-30 July 2005

Trinity College, Dublin, Ireland

\footnotetext{
* Speaker.
} 


\section{Introduction}

There is a large class of nonperturbative results or conjectured results for supersymmetric theories, particularly supersymmetric Yang-Mills theories, in 3-D [1, 2, 3]. It would be very helpful if we were able to test some of the techniques for studying SUSY theories by "solving" the theories involved in a non-perturbative way. The lattice is the best candidate method for solving such theories. Unfortunately, the lattice regulator almost inevitably breaks the SUSY. The lattice is, after all, a regularization scheme designed to preserve exact gauge symmetry at the expense of manifest Poincaré invariance and SUSY is an extension of the Poincaré group.

It is certainly possible to use the lattice to study a theory which possesses a symmetry broken by the lattice action. For instance, QCD possesses $O(4)$ invariance, broken by the lattice, but the lattice is still used to study QCD. Unfortunately, for SUSY theories containing scalars, the scalar mass term is low dimensional and breaks the SUSY. Therefore very fine tuning of the lattice action is generally required to recover SUSY in the infrared. A great deal of work has gone into looking for a way to preserve SUSY, at least in the small lattice spacing limit. Some very recent work, much of which has been presented at this conference, includes [4, 5, 6]. Good reviews exist of older work, including [7, 8], and more extensive reference lists can be found therein.

We argue here that, for all the 3-D theories of interest with $N=2$, one can proceed by actually doing the fine tuning on a conventional lattice action, the Wilson action with Wilson fermions, because these theories are super-renormalizable, such that loop corrections involving the ultraviolet converge in powers of the lattice spacing and the tuning only requires a lattice perturbation theory calculation to finite loop order. Further, we explicitly do this calculation for the case of SYM theory plus fundamental matter. This holds out the possibility of testing some very interesting claims $[2,3]$ of exact results in $N=2$ SYM theory with matter.

\section{Super-renormalizability and SUSY breaking}

Typically it is easy to write down a lattice gauge theory which, at tree level, will look in the infrared like the theory of interest. The problem is that, in the UV (at the lattice spacing scale), the lattice theory typically does not have the full symmetries of the theory we are interested in. Generally it is possible to formulate lattice theories so that they have exact gauge and (hyper)cubic symmetries. However, under SUSY, the variation of a fermionic field can involve the derivative of a bosonic field; and since derivatives become finite differences on the lattice, SUSY will generically be badly broken at the lattice spacing scale. Therefore, if we construct the lattice theory to satisfy supersymmetric relations in the infrared, radiative effects involving UV (SUSY breaking) modes will typically communicate those effects to the infrared modes of interest.

The IR effective theory is the theory one obtains, by writing down the most general continuum quantum field theory consistent with the field content and symmetries of the lattice, and performing a matching calculation between the lattice theory and that continuum effective theory, to determine what the actual parameters of the IR effective theory are. For instance, if we made a tree level lattice implementation of the Wess-Zumino model,

$$
\mathscr{L}_{\text {bare }}=\partial_{\mu} \Phi^{*} \partial^{\mu} \Phi+\psi^{\dagger} \partial \psi+\left(\lambda \Phi \psi^{\top} e \psi+\text { h.c. }\right)+\lambda^{2}\left(\Phi^{\dagger} \Phi\right)^{2},
$$


with $\Phi$ a complex scalar and $\psi$ a two component spinor, then we would generically recover an infrared theory where all terms permissible with this field content were present;

$$
\mathscr{L}_{\mathrm{IR}}=Z_{\phi} \partial_{\mu} \Phi^{*} \partial^{\mu} \Phi+Z_{\psi} \psi^{\dagger} \partial \psi+m_{\phi}^{2} \Phi^{*} \Phi+m_{\psi} \psi^{\dagger} \psi+\left(\lambda_{y} \Phi \psi^{\top} e \psi+\text { h.c. }\right)+\lambda_{s}^{2}\left(\Phi^{*} \Phi\right)^{2}
$$

plus irrelevant high dimension operators. The point is that the IR behavior typically involves radiatively generated terms which do not respect the SUSY. In particular one does not expect $m_{\psi}^{2}=m_{\phi}^{2}$. In 4-D this problem is severe. Additive scalar mass renormalizations are divergently large at every loop order; a severe nonperturbative tuning is needed to remove them.

The beauty of 3-D is that the desired theory is generally super-renormalizable. Consequently, the UV is very weakly coupled; specifically, as the lattice spacing is taken to zero, the coupling at the lattice scale falls linearly with $a$. This means that, while the SUSY breaking nature of the UV regulator radiatively induces SUSY breaking effects in the IR, the matching calculation which determines them converges very quickly. At each loop order, we determine the matching of parameters to one more power of the lattice spacing $a$. In the above model, if we compute the mass squared for the scalar field, generated by UV physics, the contributions at different orders in the loopwise expansion are again of order $\lambda^{2}, \lambda^{4}, \lambda^{6}, \ldots$ But $\lambda^{2}$ has mass dimension 1 . Since the matching calculation involves only UV physics, the only scale which can balance the explicit powers of mass is the lattice spacing scale. Therefore, the terms in the loopwise expansion are of order $\lambda^{2} / a$ at one loop, $\lambda^{4}$ at two loops and $a \lambda^{6}$ at three loops. The one and two loop contributions are significant and must be removed by an appropriate counterterm. However, three and higher loop effects vanish in the $a \rightarrow 0$ limit, and so can be neglected. For the scalar self-coupling $\lambda_{s}^{2}$, the one loop correction is already $O\left(a \lambda^{4}\right)$, and so a tree level treatment is already sufficient.

We should stress here that the infrared cancellation which ensures that only the scale $a$ appears to balance the explicit mass dimensions in the coupling, is a generic property of matching calculations in effective field theory and contains no statement about the IR physics of SUSY. Infrared divergences arise from large length scale (or low loop momentum) behavior. By construction the two theories for which any matching in an effective field theory formulation is being performed have the same IR behavior, and so any IR divergence will cancel in the difference. The statement is then simply that the IR extension of the lattice theory does describe the SUSY theory of interest provided that it contains the same degrees of freedom and that the coefficients of the terms in the Lagrangian can be matched with those of the theory of interest.

In order to perform the perturbative matching calculation analytically, and tune the lattice theory to ensure that the IR effective theory satisfies all relations implied by SUSY up to $a$ suppressed corrections, we need only compute two correlation functions, which must be sensitive to the scalar and fermionic masses, to conduct the matching calculation. The obvious candidates are the respective two-point functions (self-energies). Further, we already know that the SUSY values vanish identically. Therefore, all that is required is to compute the lattice fermionic self-energy at zero momentum, at one loop, and the lattice scalar self-energy at zero momentum, at two loops, and to assign counterterms to cancel these contributions. Therefore, the matching calculation will consist of computing a handful of IR finite linear combinations of lattice regulated Feynman graphs involving scalars, fermions, and gauge fields. 


\section{Detailed treatment of the Wess-Zumino model}

Consider a theory of scalars and fermions with $N=2$ SUSY in 3-D. The matter content is the same as for a 4-D $N=1$ SUSY theory. To make the extension to gauge theories simpler, we consider a theory with a global $\mathrm{SU}\left(N_{\mathrm{c}}\right)$ symmetry, and fields in the fundamental, $\Phi_{f}, \psi_{f}$, antifundamental, $\Phi_{a}, \psi_{a}$ and singlet representations, $\Phi_{s}, \psi_{s}$. We consider the most general superpotential. We exclude mass terms because including them will not change the mass counterterms we will need (any loopwise correction involving a mass term will be $O\left(m^{2} \lambda^{2} a\right)$ at worst).

We have chosen to implement fermions on the lattice using the Wilson action and will present results for two values of the Wilson parameter, $r=1$ and $r=\frac{1}{2}$. Our results are presented as expressions for the mass (counter)terms that are added to the Lagrangian in the obvious way. Multiplicative renormalization of couplings and wave functions is not needed at the order of interest.

We need to do three lattice Feynman integrals to complete the matching calculation, see [9] for details. We can readily verify that, at low momenta, each integrand is well behaved. None of the integrals can be done in closed form (to our knowledge), but all are relatively tractable by quadratures; we find numerically,

$$
\begin{array}{ll}
C_{y s}(r=1)=6.4706034146527591308 & C_{y s}(r=0.5)=5.057247581039541 \\
C_{y f}(r=1)=2.29977456857632 & C_{y f}(r=0.5)=2.22804716126902 \\
C_{y y}(r=1)=5.425954134(5) & C_{y y}(r=0.5)=6.8513618(8) .
\end{array}
$$

In terms of these coefficients, the required renormalizations of the masses are

$$
\begin{array}{ll}
\delta m_{s, i j}^{2}=\left(-d_{\mathrm{f}} \lambda_{l m i}^{*} \lambda_{l m j}-\frac{1}{2} \xi_{i l m}^{*} \xi_{j l m}\right) \frac{C_{y s}}{4 \pi a}, & \delta m_{f, i j}^{2}=-\lambda_{i l m}^{*} \lambda_{j l m} \frac{C_{y s}}{4 \pi a}, \\
\delta m_{a, i j}^{2}=-\lambda_{l i m}^{*} \lambda_{l j m} \frac{C_{y s}}{4 \pi a}, & \delta M_{f, i j}=\lambda_{i l m}^{*} \lambda_{j l m} \frac{C_{y f}}{4 \pi}, \\
\delta M_{s, i j}=\left(d_{\mathrm{f}} \lambda_{l m i}^{*} \lambda_{l m j}+\frac{1}{2} \xi_{i l m}^{*} \xi_{j l m}\right) \frac{C_{y f}}{4 \pi}, & \delta M_{a, i j}=\lambda_{l i m}^{*} \lambda_{l j m} \frac{C_{y f}}{4 \pi},
\end{array}
$$

at one loop and

$$
\begin{aligned}
& \delta m_{s, i j}^{2}=\left\{d_{\mathrm{f}} \lambda_{n m i}^{*} \lambda_{n q j} \lambda_{l q k}^{*} \lambda_{l m k}+d_{\mathrm{f}} \lambda_{m n i}^{*} \lambda_{q n j} \lambda_{q l k}^{*} \lambda_{m l k}+d_{\mathrm{f}} \xi_{q n i}^{*} \xi_{q m j} \lambda_{k l m}^{*} \lambda_{k l n}+\frac{1}{2} \xi_{k m i}^{*} \xi_{l m j} \xi_{k n q}^{*} \xi_{l n q}\right\} \frac{C_{y y}}{16 \pi^{2}}, \\
& \delta m_{f, i j}^{2}=\left\{\lambda_{i m n}^{*} \lambda_{j q n} \lambda_{k q l}^{*} \lambda_{k m l}+d_{\mathrm{f}} \lambda_{i n m}^{*} \lambda_{j n q} \lambda_{k l q}^{*} \lambda_{k l m}+\frac{1}{2} \lambda_{i k l}^{*} \lambda_{j k m} \xi_{n q m}^{*} \xi_{n q l}\right\} \frac{C_{y y}}{16 \pi^{2}}, \\
& \delta m_{a, i j}^{2}=\left\{\lambda_{m i n}^{*} \lambda_{q j n} \lambda_{q k l}^{*} \lambda_{m k l}+d_{\mathrm{f}} \lambda_{\text {nim }}^{*} \lambda_{n j q} \lambda_{l k q}^{*} \lambda_{l k m}+\frac{1}{2} \lambda_{k i l}^{*} \lambda_{k j m} \xi_{n q m}^{*} \xi_{n q l}\right\} \frac{C_{y y}}{16 \pi^{2}},
\end{aligned}
$$

at two loops. Here $d_{\mathrm{f}}$ is the dimension of the fundamental representation.

\section{4. $N=2 \mathbf{S U}\left(N_{\mathrm{c}}\right)$ gauge theories with fundamental matter}

Now we extend these results to the case where gauge interactions are also present. The added fields are a gauge field $A_{\mu}$, an adjoint fermionic gaugino $\chi$, and an adjoint scalar field we will write $\phi$. In the approach where we obtain a 3-D $N=2$ supersymmetric theory by dimensional reduction 
of a 4-D, $N=1$ SUSY theory, the scalar $\phi$ is the gauge field component in the direction which was compactified. Besides their gauge interactions, these fields also introduce new Yukawa and scalar interactions, which are local and can be implemented in the obvious way.

Though the matching calculation as a whole is perfectly IR safe and gauge invariant, individual diagrams are, in general, not. Each diagram will include a prefactor involving some combination of the group invariants $\left(\mathscr{T}_{F}\right.$ and $\left.\mathscr{C}_{F, A}\right)$, the Yukawa couplings, $d_{\mathrm{f}}$ and $n_{f}+n_{a}$. Since the matching calculation is valid for any Lie group, we can factorize the diagrams based on this prefactor. New counterterms are needed, which we name $\frac{1}{2} \delta m_{\phi}^{2}$ and $\delta M_{\chi}$; they have the obvious definitions. The new contributions to the one loop mass counterterms are,

$$
\begin{aligned}
& \left(\delta m_{a, f}^{2}\right)_{a b}=-2 g^{2} \mathscr{C}_{F} \delta_{a b} \frac{C_{y s}}{4 \pi a}, \quad\left(\delta m_{\phi}^{2}\right)_{A B}=-g^{2}\left(\left(n_{f}+n_{a}\right) \mathscr{T}_{F}+\mathscr{C}_{A}\right) \delta_{A B} \frac{C_{y s}}{4 \pi a} \\
& \left(\delta M_{a, f}\right)_{a b}=g^{2} \mathscr{C}_{F} \delta_{a b} \frac{C_{g f}}{4 \pi}, \quad\left(\delta M_{\chi}\right)_{A B}=g^{2} \mathscr{C}_{A} \frac{C_{g f}}{4 \pi}+g^{2}\left(\left(n_{f}+n_{a}\right) \mathscr{T}_{F}+\mathscr{C}_{A}\right) \delta_{A B} \frac{C_{y f}}{4 \pi}
\end{aligned}
$$

The new two loop counterterms are

$$
\begin{aligned}
\delta m_{s, i j}^{2}= & g^{2} \lambda_{l m i}^{*} \lambda_{l m j} \mathscr{C}_{F} d_{F} \frac{C_{g}^{\text {sing }}}{16 \pi^{2}}, \\
\left(\delta m_{a, f ; i j}^{2}\right)_{a b}= & g^{2} \delta_{a b} \lambda_{i l m}^{*} \lambda_{j l m} \mathscr{C}_{F} \frac{C_{g 1}^{f \text { und }}}{16 \pi^{2}}+g^{4} \delta_{i j} \delta_{a b}\left\{\mathscr{T}_{F} \mathscr{C}_{F}\left(n_{f}+n_{a}\right) \frac{C_{g 2}^{f u n d}}{16 \pi^{2}}+\mathscr{C}_{F}^{2}\left(\frac{C_{g 3}^{f u n d}}{16 \pi^{2}}-\frac{1}{3} \frac{\Sigma^{2}}{16 \pi^{2}}\right)\right. \\
& \left.+\mathscr{C}_{F} \mathscr{C}_{A}\left(\frac{C_{g 4}^{\text {fund }}}{16 \pi^{2}}+\frac{1}{18} \frac{\Sigma^{2}}{16 \pi^{2}}\right)-\frac{4}{3} \mathscr{T}_{F} \mathscr{C}_{F}\left(\mathscr{C}_{F}-\frac{1}{6} \mathscr{C}_{A}\right) \frac{(4 \pi \Sigma)}{16 \pi^{2}}\right\} \\
\left(\delta m_{\phi}^{2}\right)_{A B}= & g^{2} \delta_{A B} \lambda_{i j k}^{*} \lambda_{i j k} \mathscr{T}_{F} \frac{C_{g 1}^{a d j}}{16 \pi^{2}}+g^{4} \delta_{A B}\left\{\mathscr{T}_{F} \mathscr{C}_{F}\left(n_{f}+n_{a}\right) \frac{C_{g 2}^{a d j}}{16 \pi^{2}}+\mathscr{T}_{F} \mathscr{C}_{A} \frac{C_{g 3}^{a d j}}{16 \pi^{2}}\right. \\
& \left.+\left(\mathscr{C}_{A}\right)^{2}\left(\frac{C_{g 4}^{a d j}}{16 \pi^{2}}-\frac{5}{18} \frac{\Sigma^{2}}{16 \pi^{2}}\right)-\frac{4}{3} \mathscr{T}_{F} \mathscr{C}_{A}\left(\mathscr{C}_{F}-\frac{1}{6} \mathscr{C}_{A}\right) \frac{(4 \pi \Sigma)}{16 \pi^{2}}\right\}
\end{aligned}
$$

The constants appearing in these expressions are

$$
\begin{array}{ll}
C_{g f}(r=1)=-\Sigma / 2 & C_{g f}(r=0.5)=.097938749331668 \\
C_{g}^{\text {sing }}(r=1)=3.588328893(6) & C_{g}^{\text {sing }}(r=0.5)=17.8901895(7) \\
C_{g 1}^{\text {fund }}(r=1)=-4.89236097(1) & C_{g 1}^{\text {fund }}(r=0.5)=3.648535(2) \\
C_{g 2}^{\text {fund }}(r=1)=10.2296763(2) & C_{g 2}^{\text {fund }}(r=0.5)=13.32776(1) \\
C_{g 3}^{\text {fund }}(r=1)=22.712647140(8) & C_{g 3}^{\text {fund }}(r=0.5)=29.816565(2) \\
C_{g 4}^{\text {fund }}(r=1)=-2.647013(1) & C_{g 4}^{\text {fund }}(r=0.5)=9.051300(7) \\
C_{g 1}^{\text {adj }}(r=1)=7.75588650(2) & C_{g 1}^{\text {adj }}(r=0.5)=14.526578(3) \\
C_{g 2}^{\text {adj }}(r=1)=17.536258926(7) & C_{g 2}^{\text {adj }}(r=0.5)=30.769058(1) \\
C_{g 3}^{\text {adj }}(r=1)=-.3347923(2) & C_{g 3}^{\text {adj }}(r=0.5)=.76791(1) \\
C_{g 4}^{\text {adj }}(r=1)=13.0938429(1) & C_{g 4}^{\text {adj }}(r=0.5)=20.658655(8),
\end{array}
$$

where $\Sigma=3.17591153562522$. The error in each constant has been determined conservatively. 


\section{Conclusion}

There are great technical challenges encountered in the attempt to formulate lattice theories that can reproduce SUSY in the IR. The direction of the field has generally been to try to build infrared SUSY behavior into the construction of the action. We have argued that, for 3-D theories, it is feasible and straightforward instead to use the simplest possible action and perform the requisite fine tuning of its parameters analytically. This does not prove as difficult as one might have feared.

We have performed these tunings for a class of theories displaying $N=2$ SUSY in 3-D and containing arbitrary numbers of matter multiplets transforming in the fundamental representation of the gauge group. The technique is robust in the sense that it relies only on theoretical principles, like Wilson's effective action formulation, and lattice implementations, like the Wilson action for fermions, that have been rigorously studied for decades. More generally, the entire lattice action is the most simple such construction with the appropriate IR limit, such that the many complications that can arise in such theories are suitably manageable or altogether absent.

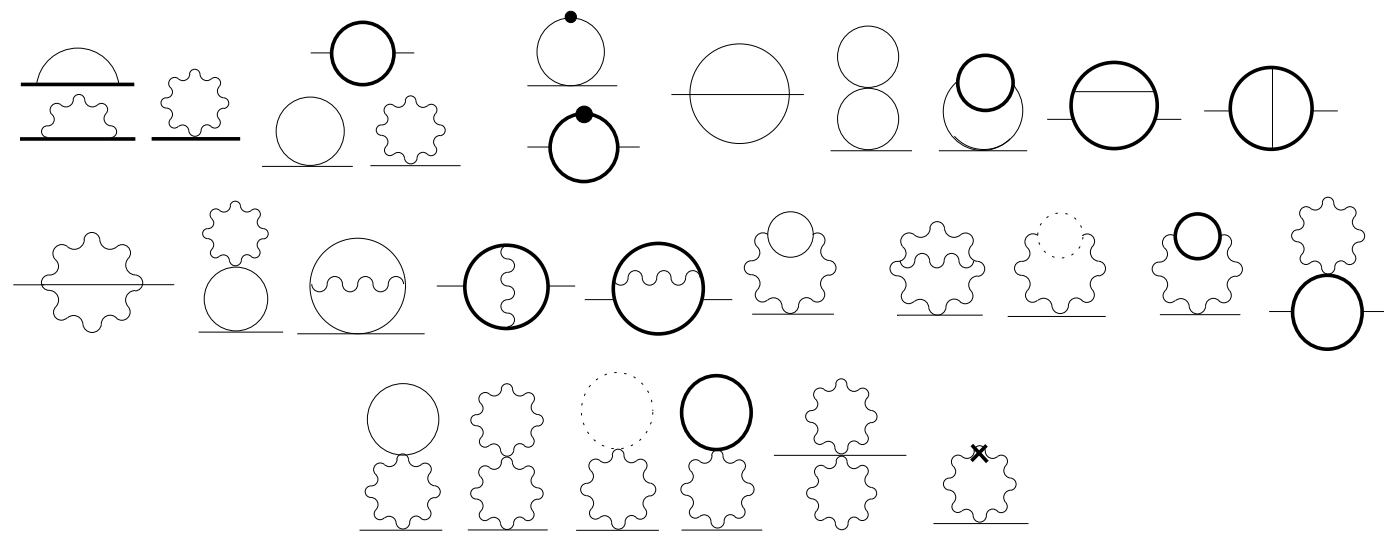

Figure 1: Diagrams needed for the renormalization of super QCD with matter fields.

\section{References}

[1] K. A. Intriligator and N. Seiberg, Phys. Lett. B 387, 513 (1996) [hep-th/9607207].

[2] O. Aharony, A. Hanany, K. A. Intriligator, N. Seiberg and M. J. Strassler, Nucl. Phys. B 499, 67 (1997) [hep-th/9703110].

[3] J. de Boer, K. Hori and Y. Oz, Nucl. Phys. B 500, 163 (1997) [hep-th/9703100].

[4] D. B. Kaplan and M. Unsal, arXiv:hep-lat/0503039.

[5] S. Catterall, JHEP 0506, 027 (2005) [arXiv:hep-lat/0503036].

[6] M. Bonini and A. Feo, Phys. Rev. D 71, 114512 (2005) [arXiv:hep-lat/0504010].

[7] A. Feo, Mod. Phys. Lett. A 19, 2387 (2004) [arXiv:hep-lat/0410012].

[8] D. B. Kaplan, Nucl. Phys. Proc. Suppl. 129, 109 (2004) [arXiv:hep-lat/0309099].

[9] J. W. Elliott and G. D. Moore, arXiv:hep-lat/0509032.

[10] H. J. Rothe, "Lattice gauge theories: an introduction,” World Sci. Lect. Notes Phys. 59, 1 (1997). 